\title{
Underrecognition of Sleep Disorders in Patients with Multiple Sclerosis
}

\author{
Tiffany J. Braley, MD, MS 1,$2 ;$ Benjamin M. Segal, MD²; Ronald D. Chervin, MD, MS ${ }^{1}$ \\ ${ }^{1}$ University of Michigan Department of Neurology and Sleep Disorders Center, Ann Arbor, MI; ${ }^{2}$ University of Michigan Department \\ of Neurology and Multiple Sclerosis Center, Ann Arbor, MI
}

W e were pleased to read the recent article by Brass et al. entitled "The Underdiagnosis of Sleep Disorders in Patients with Multiple Sclerosis," published in the September 2014 issue of Journal of Clinical Sleep Medicine. ${ }^{1}$ This well-conceived study strongly supports recent evidence that MS patients are at increased risk for sleep disturbances, and suggests that all clinicians who treat patients with MS should routinely screen these patients for sleep disorders.

It is noteworthy that the findings by Brass et al. complement recently published findings from our center, which reported the results of a comprehensive sleep survey among 195 patients with a confirmed diagnosis of MS. ${ }^{2}$ Our survey included many of the validated measures used in the Brass study (STOP-Bang Questionnaire, Insomnia Severity Index [ISI], items adapted from the International Restless Legs Syndrome [RLS] Study Group diagnostic criteria, and Fatigue Severity Scale), as well as similarly structured questions regarding sleep duration, latency, and number of nocturnal awakenings. Forty-six percent of subjects met ISI criteria for moderate or severe clinical insomnia, and 30\% met criteria for RLS. We also demonstrated a large discrepancy between OSA risk and diagnosis - $21 \%$ of MS patients carried a formal diagnosis of OSA, while $56 \%$ were at elevated risk for OSA based on the STOP-Bang. More importantly, OSA risk emerged as a significant predictor of fatigue, after adjustment for other clinical and sleep-related confounders (confirmed by medical chart review).

While the majority of results were similar between studies, variability in estimates of elevated OSA risk (38\% vs. 56\%) and OSA diagnosis ( $4 \%$ vs. $21 \%$ ) deserve comment, and may reflect differences in subject populations. A significant strength of the Brass study was the utilization of a large, population-based sample, while strengths of our study included confirmation of MS diagnosis and consideration of clinically relevant confounders that could influence study results. As acknowledged by the authors, response bias could have led to underestimations of OSA risk or prevalence in the Brass study, particularly if healthier or less disabled patients represented the majority of responders. Conversely, as our study surveyed patients in a tertiary care center, it is possible that referral bias could in part explain our higher estimates of OSA risk and prevalence. Geographical differences in practice patterns could also influence outcomes. Nonetheless, both studies suggest a strikingly high prevalence of sleep disturbances among patients with MS and highlight a significant gap between risk and recognition of these conditions. Increased efforts are needed to facilitate early recognition and treatment of sleep disorders among persons with MS, and we hope that these collective data will influence the medical community at large to implement appropriate screening measures in their clinical practice.

\section{CITATION}

Braley TJ, Segal BM, Chervin RD. Underrecognition of sleep disorders in patients with multiple sclerosis. J Clin Sleep Med 2015;11(1):81.

\section{REFERENCES}

1. Brass SD, Li CS, Auerbach $S$. The underdiagnosis of sleep disorders in patients with multiple sclerosis. J Clin Sleep Med 2014;10:1025-31.

2. Braley TJ, Segal BM, Chervin RD. Obstructive sleep apnea and fatigue in patients with multiple sclerosis. J Clin Sleep Med 2014;10:155-62.

\section{SUBMISSION \& CORRESPONDENCE INFORMATION}

\section{Submitted for publication October, 2014} Accepted for publication October, 2014

Address correspondence to: Tiffany J. Braley, MD, MS, Assistant Professor of Neurology, University of Michigan Department of Neurology, C728 Med-Inn Building, 1500 E. Medical Center Dr.,Ann Arbor, MI 48109; Tel: (734) 232-1147; Fax: (734) 232-9986; Email: tbraley@med.umich.edu

\section{DISCLOSURE STATEMENT}

Dr. Braley has served as site P.I. on several industry-sponsored studies at the University of Michigan, including studies sponsored by Sanofi-Genzyme, GenentechRoche, Biogen-Idec, and AB Science but received no direct compensation for this work, and these studies were not related to the research presented in this manuscript. She is the recipient of an American Sleep Medicine Foundation Bridge-to-K grant, awarded in 2012. She is also named in a provisional patent, held by the University of Michigan, concerning treatment for obstructive sleep apnea. Dr. Segal is PI for an investigator initiated educational grant from TEVA to support an academic lecture series, has received research support from Therakos, serves as mentor for an MS clinical fellowship grant sponsored by Biogen-ldec, and has given grand rounds lecture for Novartis. He has served as a consultant for Novartis and Biogen-ldec. He is also named in a provisional patent, held by the University of Michigan, concerning treatment for obstructive sleep apnea. Dr. Chervin has received NIH, research grants; University of Michigan, research grants; Philips Respironics and Fisher Paykel, support to University of Michigan for educational program in sleep biomedical innovation; Board of Directors, American Academy of Sleep Medicine, American Sleep Medicine Foundation, American Board of Sleep Medicine, Associated Professional Sleep Societies, International Pediatric Sleep Association; Advisory Board (volunteer), Sweet Dreamzzz (not-for-profit community organization); Proctor \& Gamble, consultant, through contract established with University of Michigan; MC3, consultant; Zansors, consultant, (not compensated); Zansors, licensed questionnaire (developed by author) from University of Michigan; UpToDate, editor; Cambridge University Press, book editor; Named in copyrighted material and patents, held by the University of Michigan, for sleep apnea and sleep disorder-related diagnosis, assessment, and treatments. 